\title{
Regulation of autophagy by microRNAs in human breast cancer
}

\author{
Zhi Xiong Chong ${ }^{1}$, Swee Keong Yeap ${ }^{2}$ and Wan Yong Ho ${ }^{1 *}$ (D
}

\begin{abstract}
Breast cancer is the most common solid cancer that affects female population globally. MicroRNAs (miRNAs) are short non-coding RNAs that can regulate post-transcriptional modification of multiple downstream genes. Autophagy is a conserved cellular catabolic activity that aims to provide nutrients and degrade un-usable macromolecules in mammalian cells. A number of in vitro, in vivo and clinical studies have reported that some miRNAs could modulate autophagy activity in human breast cancer cells, and these would influence human breast cancer progression and treatment response. Therefore, this review was aimed to discuss the roles of autophagy-regulating miRNAs in influencing breast cancer development and treatment response. The review would first introduce autophagy types and process, followed by the discussion of the roles of different miRNAs in modulating autophagy in human breast cancer, and to explore how would this miRNA-autophagy regulatory process affect the disease progression or treatment response. Lastly, the potential applications and challenges of utilizing autophagy-regulating miRNAs as breast cancer biomarkers and novel therapeutic agents would be discussed.
\end{abstract}

Keywords: Breast cancer, miRNAs, Autophagy, Biomarkers, Therapeutic agents

\section{Introduction}

Breast cancer is currently the number one cancer that affects the female population worldwide and every year, more than 2 million females will be diagnosed to have this malignancy [1]. It is estimated that more than 40,000 breast cancer patients passed away because of this malignancy in year 2019 alone [2]. The challenge in managing this heterogenous malignancy is that this cancer is highly aggressive [3], and it is always associated with problems like chemoresistance [4], radioresistance [5], resistance towards hormonal therapy [6] and resistance towards targeted therapy [7].

MicroRNAs (miRNAs) are short endogenous, singlestranded, non-coding RNAs (ncRNAs) which contain 18-25 nucleotides [8, 9]. MiRNAs have been reported to play essential roles in regulating the post-transcriptional

\footnotetext{
*Correspondence: WanYong.Ho@nottingham.edu.my

${ }^{1}$ Faculty of Science and Engineering, University of Nottingham Malaysia, 43500 Semenyih, Selangor, Malaysia
}

Full list of author information is available at the end of the article modification of multiple downstream targets [10]. By binding to the complementary sequences at the $3^{\prime}$-untranslated regions ( $3^{\prime}$-UTR) of the mRNAs of the target genes, miRNAs could repress the translation of these target genes [11]. With the advancement in the field of molecular biology and clinical science, in the past 20 years, many in vitro, in vivo and clinical studies have been conducted to investigate the potential roles of miRNAs as biomarkers in diagnosing and predicting the prognosis of human breast cancer [12, 13], and, as novel therapeutic agents to tackle breast cancer $[14,15]$.

Autophagy is a conserved, ubiquitous and important cellular degradative and catabolic activity that aims to maintain cellular homeostasis $[16,17]$. This cellular process has been described for more than 30 years ago and it started to gain enormous attention worldwide when Professor Yoshinori Ohsumi was awarded with the Nobel Prize in Medicine and Physiology in year 2016 because of his distinctive works in autophagy [18]. Dysregulation of autophagy has been reported to associate with a number of communicable [19] and non-communicable diseases 
[20, 21]. Autophagy-related non-communicable diseases can be further divided into cancerous or non-cancerous diseases like cardiovascular disease [22], neurodegenerative disease [23, 24] and skin disease [25]. Malignancies which are related to autophagy dysregulation include colorectal cancer [21], gastric cancer [26], breast cancer [27, 28], lung cancer [29], leukemia and lymphoma [30], ovarian cancer [31], and few other cancers [32,33].

Autophagy is essential to provide nutrients to the cancer cells to grow and at the same time, eliminate unusable cellular macromolecules that could potentially pose harm to the cancer cells [34]. From some published in vitro, in vivo and clinical studies' findings [35-37], regulation of autophagy by miRNAs have been demonstrated to exert some effects in influencing the human breast cancer development and treatment response. In other word, a number of miRNAs was shown to be able to either up- or down-regulate cellular autophagy, and this would eventually enhance or suppress breast cancer progression. This review, therefore, was aimed to summarize the published findings from various studies on the potential roles of miRNAs in regulating autophagy in human breast cancer, and subsequently, to discuss how does this miRNA-autophagy modulation process would influence breast cancer development and treatment response. The review would first introduce autophagy types and process, followed by the discussion on the roles of autophagy-regulating miRNAs in influencing human breast cancer development and treatment response. Lastly, the applications and challenges of employing autophagy-regulating miRNAs as potential breast cancer biomarkers and therapeutic agents would be discussed.

\section{Autophagy types and process, and functional roles of autophagy in breast cancer}

The main objective of autophagy or "self-eating" (Greek meaning) is to break down cytoplasmic components like macromolecules and organelles, in order to sustain cellular metabolism and to ensure cellular homeostasis $[16,38,39]$. Some people described autophagy as a cellular "re-cycling process" that digests old and unwanted substances and turns it into useful nutrients for cellular usage [25]. Autophagy is important to avoid the accumulation of harmful substances like precipitated proteins, damaged cellular organelles and oncogenic materials that could pose danger to the cells [38, 39]. The failures to eliminate these unnecessary, aged or toxic substances would trigger intracellular inflammation which would generate reactive oxygen species (ROS) and subsequently, these cellular processes would lead to the development of cellular degeneration, apoptosis and carcinogenesis [38-40].

\section{Types of autophagy}

Autophagy can be generally divided into three types (Fig. 1), namely, macroautophagy, microautophagy and chaperone-mediated autophagy (CMA) [16]. Macroautophagy is an evolutionarily, highly conserved and common type of autophagy that involves the sequestration of a portion of a cellular organelle to form autophagosome [41]. Lysosome then fuses with the autophagosome to form autolysosome in which the macromolecules like proteins and organelles to be degraded will be digested within the autolysosome [39]. Selective macroautophagy is a specific type of autophagy in which selected dysfunctional cellular organelles or substrates will be recognized and selected for autophagy [38]. Examples of selective macroautophagy include mitophagy (mitochondrion), lysophagy (lysosome), pexophagy (peroxisome), ribophagy (ribosome), reticulophagy (endoplasmic reticulum) and nucleophagy (nuclear components) [38]. Microautophagy is a type of autophagy in which multiple, small vesicles are engulfed by the lysosomes via lysosomal cytoplasmic invagination [16]. The detail mechanism of microautophagy in lysosome is still unclear [42] but for endosomal microautophagy, several proteins like endosomal sorting complexes required for transport I and III (ESCRT/III) and heat shock cognate $71 \mathrm{kDa}$ protein (HSC70), have been reported to play essential roles in initiating the electrostatic interaction between the protein substrates and endosomes for autophagy to happen [16]. CMA, on the other hand, is a highly selective process in which the cytoplasmic constituents that are tagged with a special C-terminal KFERQ motif will be recognized by chaperone protein like HSC70, which will then guide them to the lysosome by binding the motif to the lysosome-associated membrane protein type 2a (LAMP-A2) protein on the lysosome surface [43]. LAMP-2A is stabilized by two proteins, namely, GFAP and HSP90 [42]. LAMP-2A monomeric protein must form a multimeric complex to transport the substrate into the lysosome for degradation and this is a dynamic process which involves the monomers assembly for substrate translocation and upon completing the mission, the multimeric complex will be dissociated and degraded [16].

\section{Autophagy steps}

Next, the molecular mechanism of general autophagy process (Fig. 2) will be discussed. Cellular conditions like nutrients deprivation, presence of oxidative stress or growth hormones and accumulation of senescence organelles will generate autophagy signals to the affected cells $[16,44]$. The autophagy process can be generally divided into five stages including initiation, elongation, nucleation, fusion and degradation [39]. This cellular process 

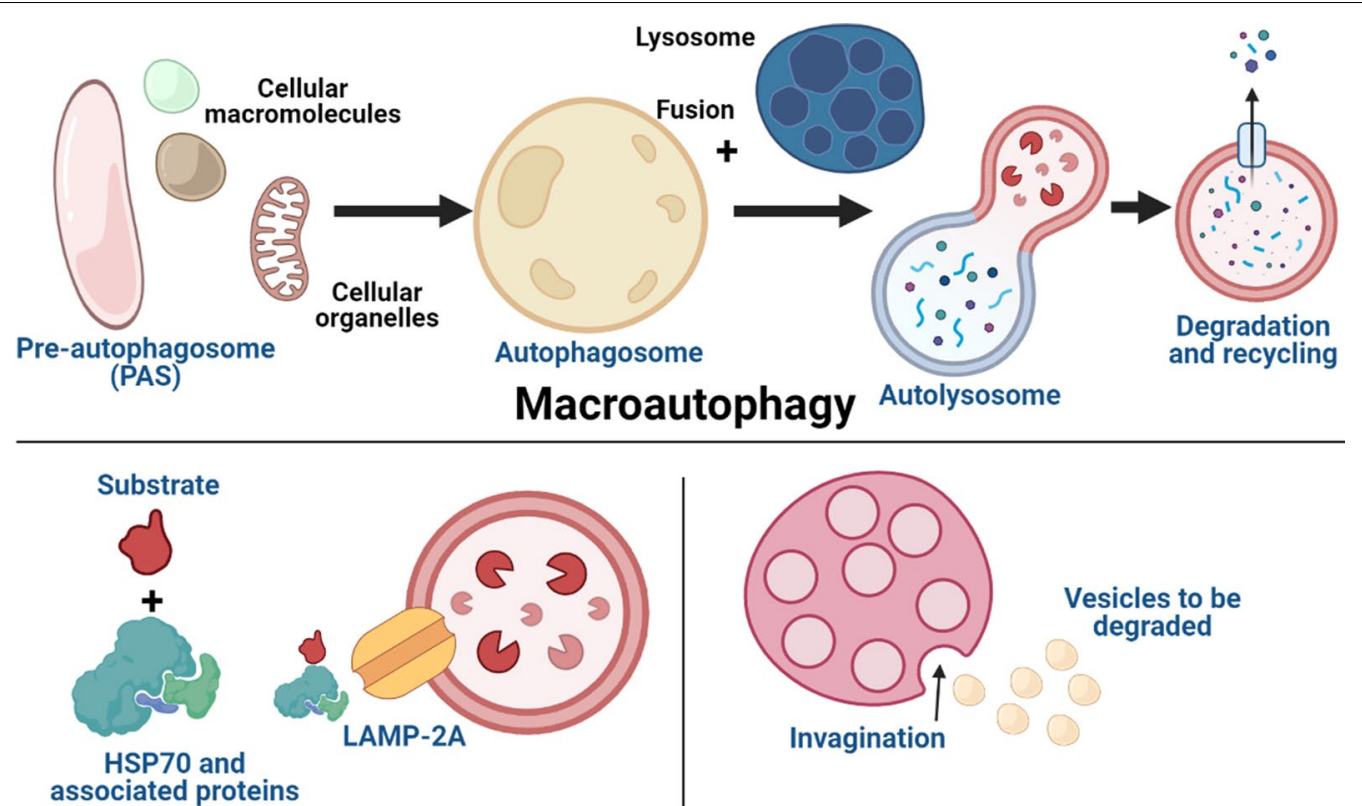

\section{Chaperon-assisted autophagy}

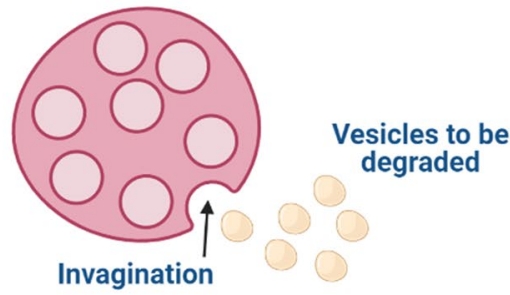

\section{Microautophagy}

Fig. 1 Types of autophagy. Autophagy can be divided into macroautophagy, microautophagy and chaperon-mediated autophagy (CMA) [16]. Macroautophagy is a highly conserved cellular process that involves the formation of autophagosome via sequestration of a portion of a cellular component to form autophagosome [39]. Fusion of autophagosome and lysosome forms autolysosome in which degradation of macromolecules and dysfunctional cellular organelles would take place [16]. Microautophagy involves the engulfment of a vesicle by lysosome via cytoplasmic invagination [42]. CMA is a type of highly selective autophagy in which only specific macromolecules that are tagged with a special C-terminal KFERQ motif will be recognized by specific chaperon protein (HSC70), this will then facilitate the binding of the selected macromolecules to the LAMP-A2 protein on the lysosome surface for autophagy to happen [43]

will begin at the endoplasmic reticulum (ER) in which part of the double layer membrane of the ER will be bud off to form a cup shape substance called pre-autophagosome (PAS) [45]. A protein complex consists of Unc-51 like autophagy activating kinase (ULK1), autophagy related proteins (Atg13/Atg101) and FAK family kinaseinteracting protein of $200 \mathrm{kDa}$ (FIP200) will be recruited and bound to the PAS [46]. This is known as the initiation step of autophagy [46]. The ULK kinase complex phosphorylates and activates autophagy and beclin-1 regulator (AMBRA) protein, and AMBRA then phosphorylates a class III phosphoinositide 3-kinase (PI3K) complex consisting of Beclin1, ATG14L, VPS34 and VPS15 [16]. The activated PI3K complex can now convert phosphatidylinositol-4,5-bisphosphate (PIP2) to phosphatidylinositol-3,4,5-bisphosphate (PIP3) [47]. The elevation of the surrounding PIP3 concentration attracts another two proteins called WD repeat domain phosphoinositide-interacting protein (WIPI2) and zinc-finger FYVE domain-containing protein 1 (DFCP1) to the PAS membrane [46]. WIPI2 protein has been reported to play essential roles in binding the ATG16L1 protein and this aids in attracting the ATG16L1/ATG5/ATG12 protein complex to the PAS [46]. The recruitment of ATG16L1/
ATG5/ATG12 complex is important in preventing the premature fusion of the autophagosome with the lysosome [16]. Besides, other proteins which are important in cargo or macromolecules sequestration like p62, sequestosome 1 (SQSTM1) and NBR1 will also be attracted and bind to the PAS [18]. SQSTM1/p62 and NBR1 are important in regulating ubiquitylation processes by facilitating the binding of the selective ubiquitinated proteins to the PAS to be removed via autophagy activity [18]. As different proteins are being recruited and bound to the PAS, it also elongates in preparation for subsequent nucleation process [39].

Once most of the macromolecules, organelles and proteins required for degradation have been recruited, a key protein that aids in the membrane closure called LC3 will come and bind to the membrane of the preautophagosome [48, 49]. LC3 is derived from pro-LC3 and the cleavage by cysteine protease ATG4 will produce LC3-I [50]. ATG3/ATG7 bound complex will then come and facilitate the binding of LC-3 with phosphatidylethanolamine (PE) to form PE-conjugated LC3-II [49]. Together with other autophagy related proteins, PE-conjugated LC3-II will initiate the membrane sealing to form a mature autophagosome $[39,49]$. Since LC3-II protein 


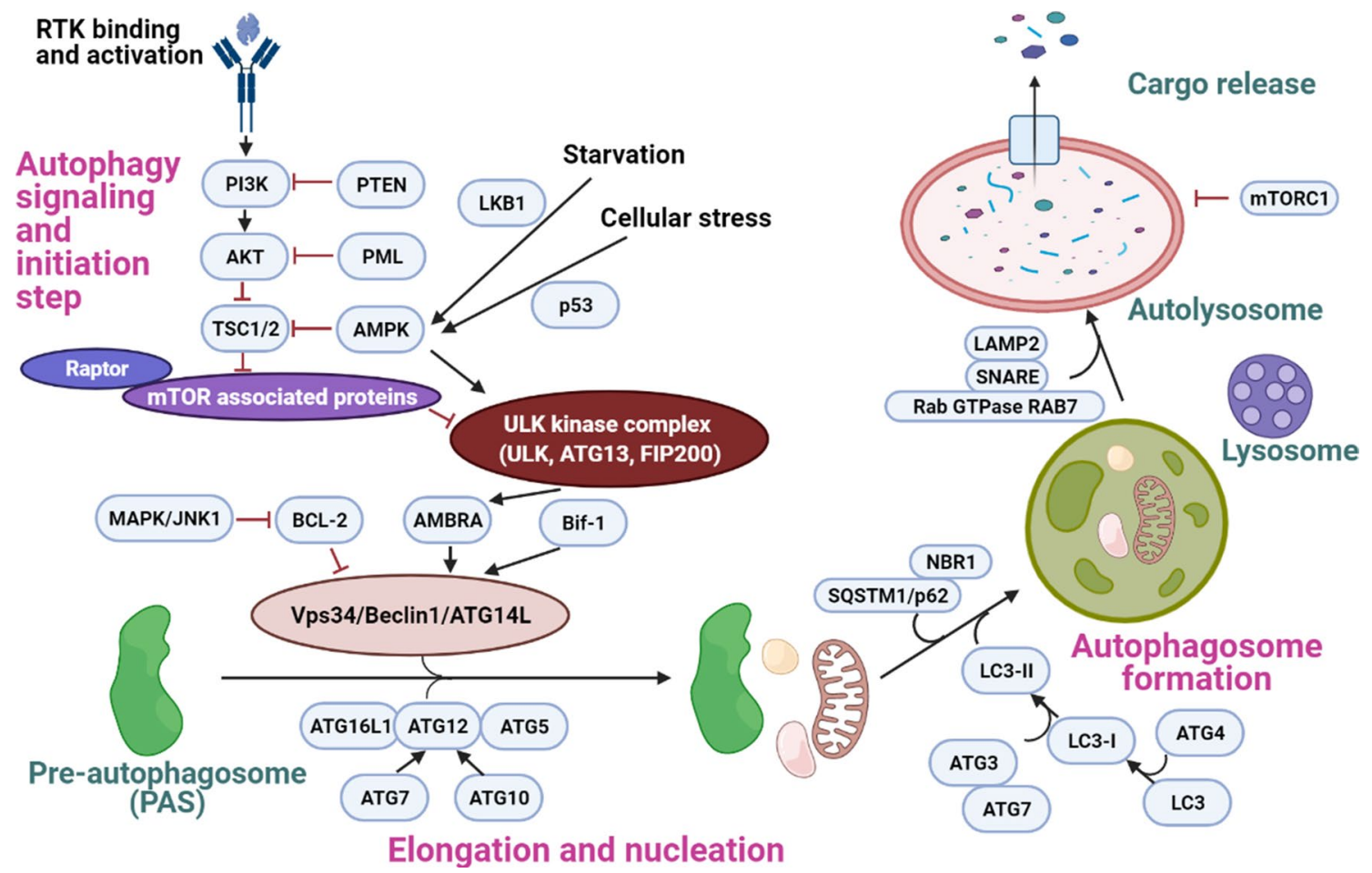

Fig. 2 Molecular mechanism of autophagy. There are generally five steps of autophagy which include initiation, elongation, nucleation, fusion and degradation [39]. Energy deprivation or cellular stress will generate cellular autophagy signals that activate the ULK1 kinase complex to activate the downstream AMBRA protein [16]. AMBRA then phosphorylates the class III PI3K to increase PIP3 level and this helps recruiting a number of autophagy related proteins like WIPI2, ATG16L1, ATG12, ATG5 and SQSTM1/p62 to the pre-autophagosome (PAS) [16]. As these essential proteins are being recruited, PAS elongates and the production of lipidated LC3-II helps the PAS to surround and enclose the substrate macromolecules to be digested [46]. When the nucleation process is completed, mature autophagosome is formed and it is ready to bind to the lysosome to form autolysosome [39]. Positive regulators of autophagy process include AMPK [54], PTEN [56], Bif-1 [58] and TFEB [59] while negative regulators of autophagy include Akt [56], mTORC1 [60] and Bcl-2 [57]

is a vital protein involved in the autophagosome formation and nucleation process, the detection of LC3-II has been regarded as one of the usual approaches to measure autophagy activity [39]. Once the membrane of the mature autophagosome is fully formed, LC3-II will be detached and now the autophagosome is ready for fusion with the lysosome [39]. Presence of other proteins likes soluble NSF attachment protein receptor (SNARE), lysosome associated membrane protein (LAMP2) and Rab GTPase RAB 7 on the mature autophagosome and lysosome surface will facilitate the fusion with the targeted lysosome to form autolysosome [46, 51]. A number of tethers (HOPS, ATG14L and TECPR1), motor adaptors (FYCO1, PLEKHM1/2 and RILP) will also be involved in mediating the fusion process [51]. As the fusion completes, the autophagosome contents will be released and these substances will be degraded in an acidic environment containing enzymes like cathepsin B, cathepsin L and other hydrolytic enzymes [52, 53]. The digested substances will then be released from the autolysosome via protein channels and it is now can be used for anabolism or other cellular processes $[16,52,53]$. The ultimate fate of the autolysosome, however, is still poorly understood, and it is unclear whether it will dissociate into lysosome and autophagosome after the digestion process is completed [39].

There are a number of proteins which have been proven to play important roles in regulating the cellular autophagy activities [16]. Energy deprivation would raise intracellular adenosine monophosphate-activated protein kinase (AMPK) level and AMPK could phosphorylate ULK1 to activate it while inhibiting tuberous sclerosis $1 / 2$ protein $(\mathrm{TSC} 1 / 2)$ to inactivate the mTOR signaling pathway that could block activation of the ULK kinase complex $[16,54]$. Protein kinase B or Akt signaling is known to inhibit autophagy induction by activating $\mathrm{mTOR}$ and the presence of PTEN will inhibit the PI3K/Akt signaling pathway [55]. As a result, inactivation of the Akt signaling pathway by PTEN will promote autophagy activity [56]. Bcl-2, an important regulating protein of the apoptotic pathway can bind and inhibit Beclin1 to prevent the formation of autophagosome [57]. Endophilin (Bif-1) is 
one of the core proteins involved in the vesicle endocytosis and it has been reported to play a vital role in interacting with Beclin-1 to regulate autophagosome formation [58]. Besides, autophagy regulation can also take place at the last phase of autophagy, which is during the autolysosome degradation process [59]. Transcription factor EB (TFEB) and mTOR complex 1 (mTORC1) are two essential proteins in coordinating the lysosome nutrient sensing machinery (LYNUS) of the lysosome [60]. Presence of abundant amino acids inside the lysosome will be sensed by vATPase, a hydrogen pump at the lysosomal membrane surface; and vATPase will relay the information to the rags proteins of the LYNUS complex [60]. When this occurs, mTORC1, which now binds to the LYNUS, will phosphorylate TFEB to inactivate it and prevent it from translocating into the nucleus [60]. Inactivation of TFEB will results in the reduction of the transcription of key genes involved in regulating endocytosis and autophagy, and downregulate the lysosomal biogenesis $[59,60]$. This prevents the formation of new autolysosome via fusion of lysosome and autophagosome [60].

\section{Functional roles of autophagy in breast cancer}

Like in other human cancer, autophagy could help in either promoting or inhibiting breast cancer [61]. A previous review has reported that autophagy plays a dynamic role in the breast cancer development, and it can be tumour-inhibiting in the early phase of cancer but become tumour-promoting in the later phase of cancer [62]. For tumour-promoting role, autophagy is an important cellular process which helps to provide nutrients and remove harmful cellular macromolecules from the breast cancer cells [63]. Thus, autophagy is said to be playing a vital role in maintaining breast cancer cells homeostasis [63], which in turn this could help the cancer cells to survive in stressful conditions like after radiotherapy or targeted therapy have been administered [63, 64]. However, autophagy can be detrimental to the breast cancer cells if it occurs excessively as autophagy is a catabolic cellular process that can lead to cellular death [65]. In some study, it has been reported that autophagy induction can be employed as one of the strategies to accelerate breast cancer cells death and to sensitize the breast cancer cells towards breast cancer therapies like chemotherapy and hormonal therapy [66]. Since autophagy is a complicated cellular process, which its role as a "friend" or "foe" in breast cancer progression is still debatable [65], more functional study is therefore needed to be conducted in the future to determine whether this cellular activity is more likely to promote or inhibit breast cancer development. Clarification of the exact role of autophagy in breast cancer development can help to strategize beneficial therapeutic options that either induce or inhibit autophagy in breast cancer patients to better eradicate this malignancy from the patients' body.

\section{Autophagy regulation by miRNAs in human breast cancer: how does this influence disease progression or treatment response}

In this review, a total of 41 miRNAs reported from 26 various in vitro, in vivo and clinical studies (Table 1) were included to discuss their roles in regulating autophagy process in human breast cancer development. In general, these autophagy-regulating miRNAs could modulate cellular autophagy (Fig. 3) by regulating (1) autophagy initiation, (2) elongation and nucleation steps moderated by autophagy-related proteins (ATGs), (3) autophagosome formation, and (4) expression of other proteins which do not involve directly in autophagy development. Out of these 41 reported miRNAs, 11 autophagy-regulating miRNAs were further reported to play vital roles in regulating treatment response in breast cancer (Table 2).

\section{Roles of miRNAs in regulating autophagy initiation}

Activation of PI3K/Akt/mTOR signaling pathway has been proven to play an essential role in downregulating cellular autophagy activity by activating mTOR protein that suppresses ULK kinase complex activity (Fig. 2) [55]. An in vitro study reported in year 2018 showed that upregulation of miR-486-5p would downregulate PTEN and this resulted in the activation of the PI3K/Akt/mTOR signaling pathway to suppress autophagy and enhance tumorigenesis [56]. PTEN is responsible in dephosphorylating PIP3, an intermediate lipid signaling molecule in the PIP3/Akt/mTOR signaling pathway, and this causes the cessation of signals along this cellular pathway [55].

ULK kinase complex comprises ULK1, ATG13, ATG101 and FIP200 proteins and it is responsible in phosphorylating the downstream Beclin-1 protein to initiate the autophagy activity [49]. In an in vitro study [67] involving triple-negative human breast cancer cell line, MDA-MB-231, the authors reported that $18 \mathrm{miR}$ NAs could play a role in downregulating the signaling hub involving ULK1 and these miRNAs include miR30c-1, miR-149, miR-611, miR-615-5p, miR-659, miR636, miR-638, miR-659, miR-675, miR-1303, miR-1308, miR-1908, miR-1914, miR-1915, miR-2861, miR-3184, miR-4292 and miR-4259. Three other miRNAs which were reported to be able to downregulate ULK1 include miR-25 [68], miR-489 [37] and miR-1275 [69]. By suppressing ULK1 expression, these 21 miRNAs could potentially block autophagy induction by reducing the activation of the AMBRA protein, in which AMBRA is important in phosphorylating the downstream Beclin-1 containing class III PI3K [16]. For miR-489, other than being shown to negatively regulate autophagy activity, 


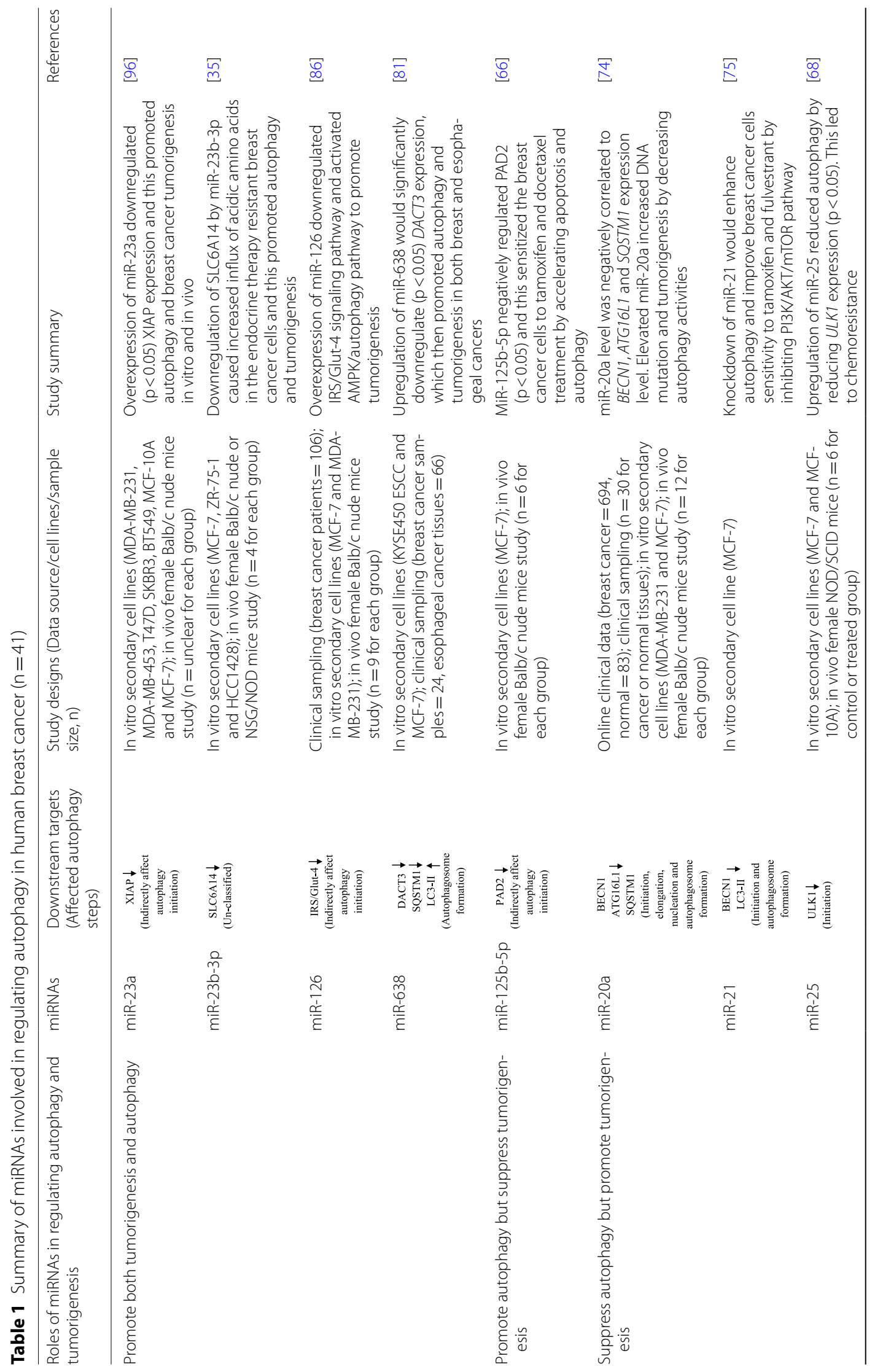




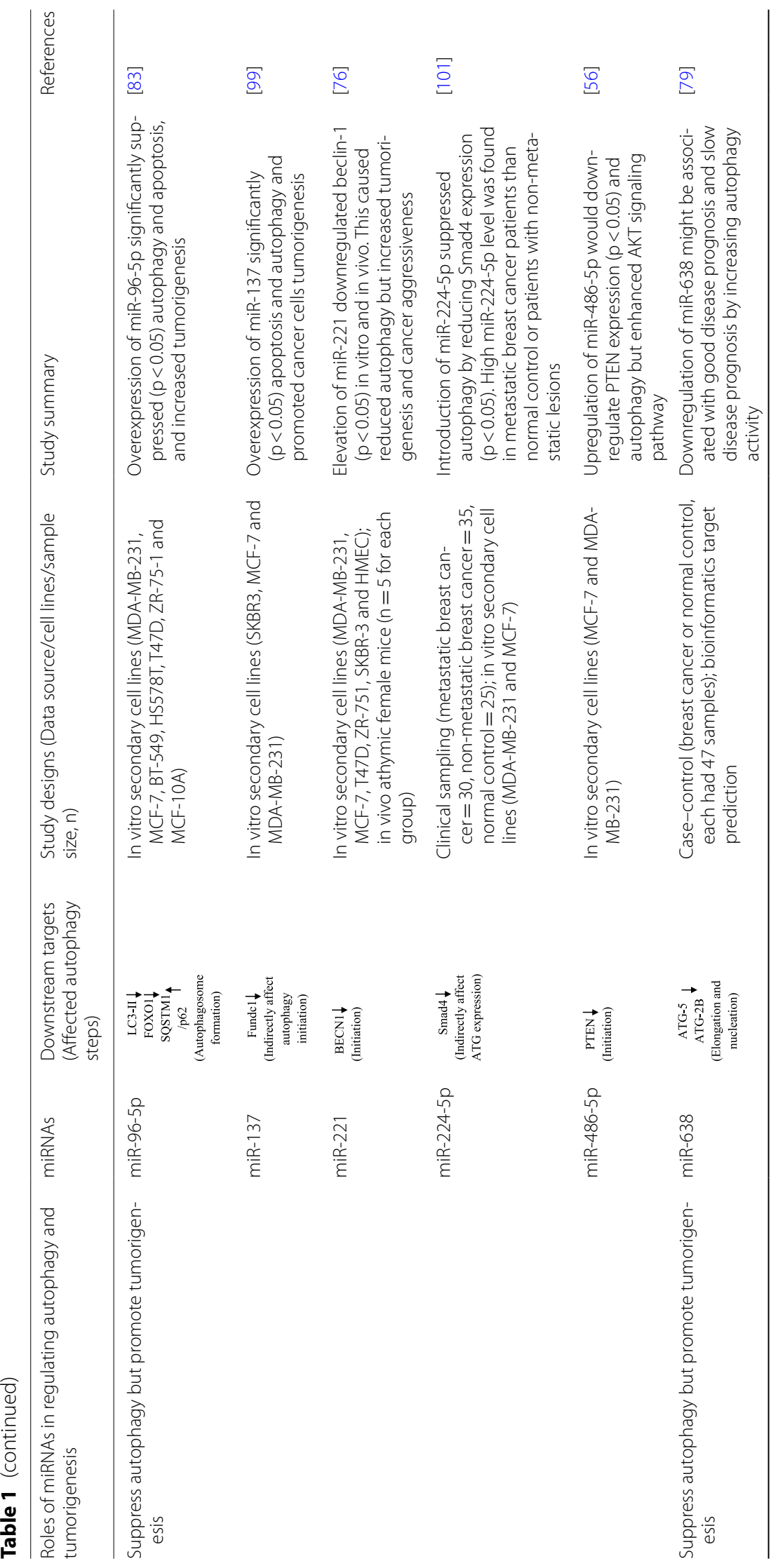




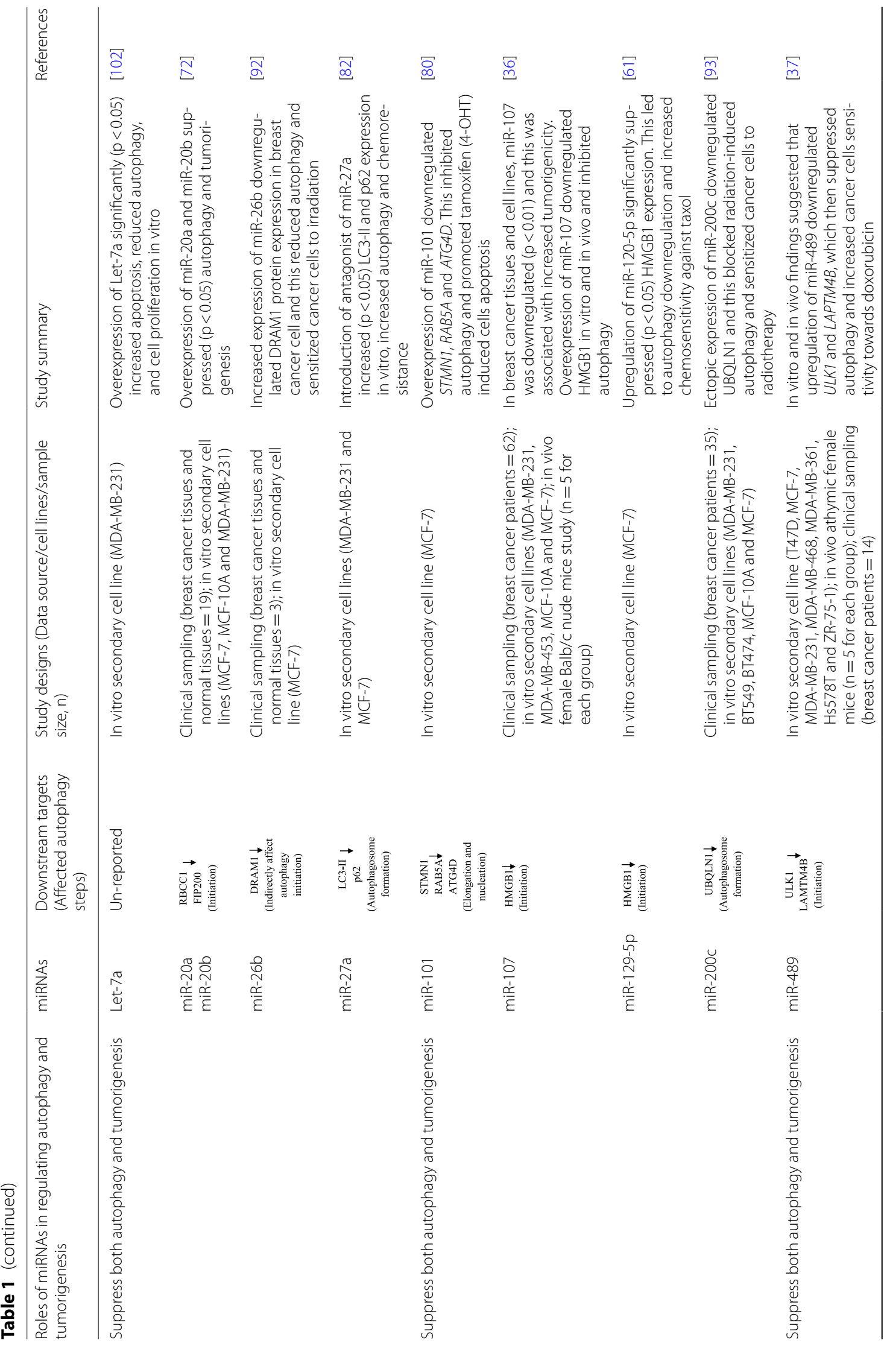




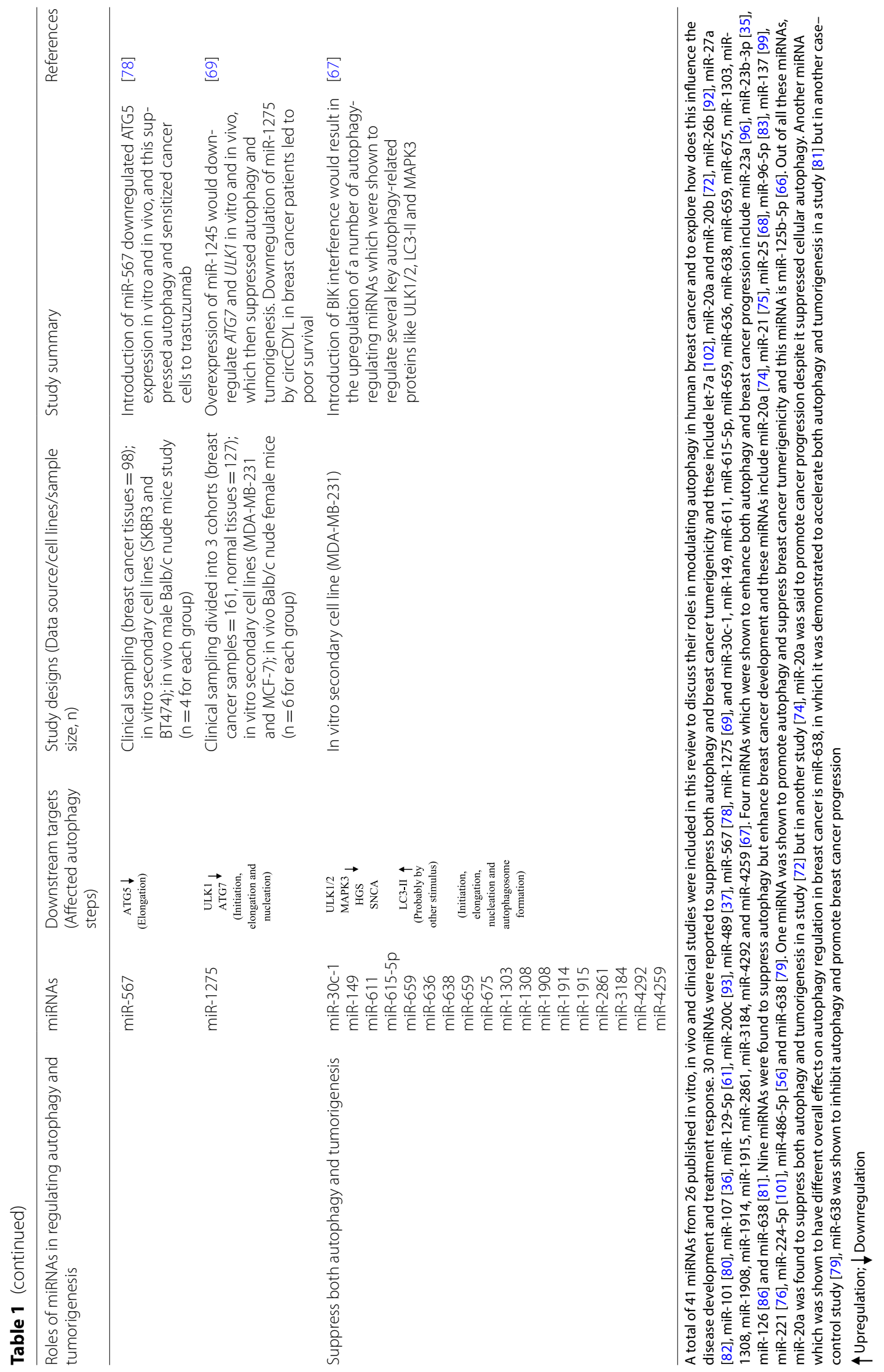




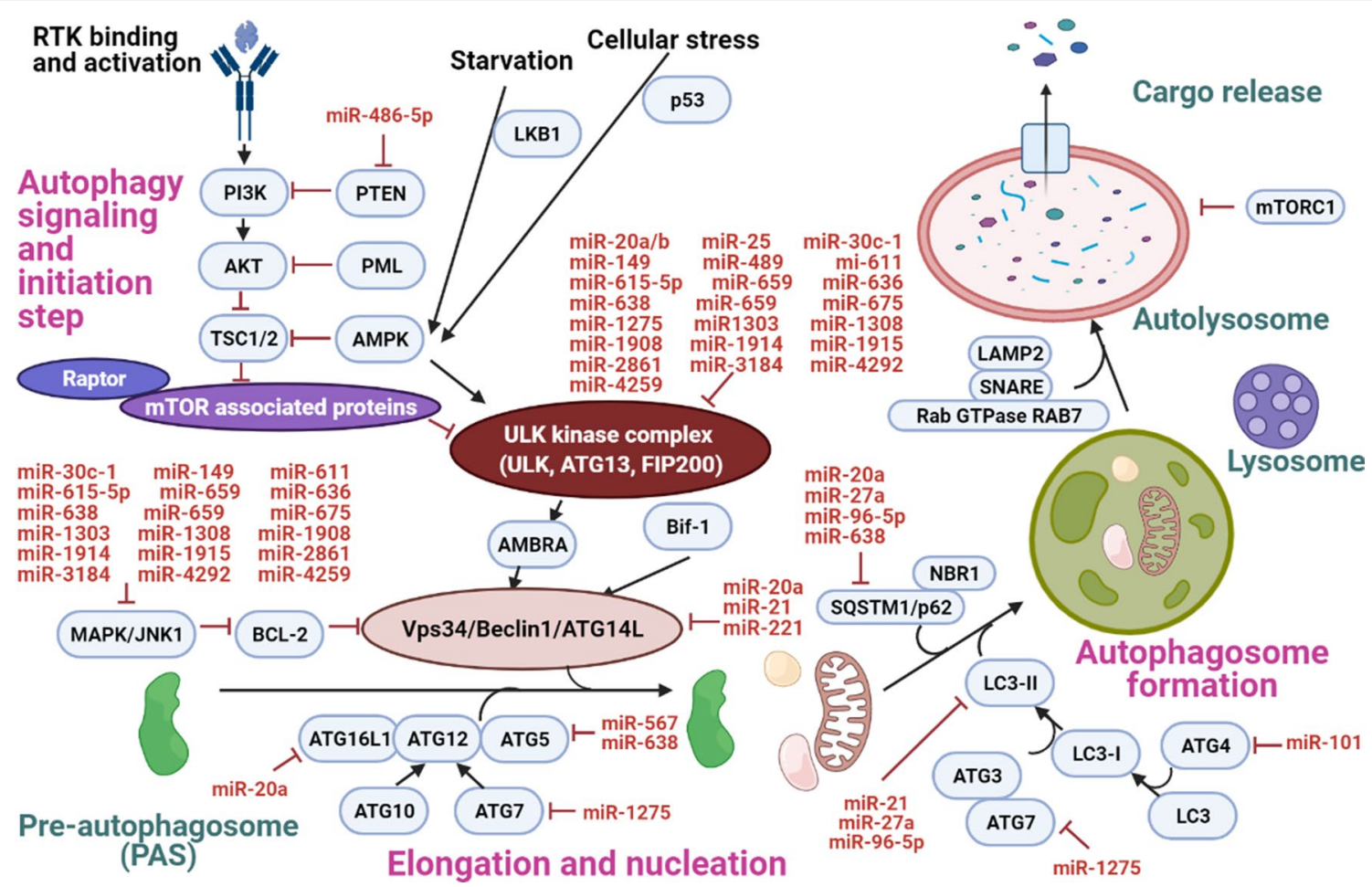

Fig. 3 Roles of miRNAs in modulating the autophagy process in human breast cancer. MiR-486-5p is said to downregulate PTEN and this activates PI3K/Akt signaling pathway to inhibit autophagy activity [56]. An in vitro study reported eighteen miRNAs were involved in downregulating the signaling hub which covers ULK1 and these miRNA include miR-30c-1, miR-149, miR-611, miR-615-5p, miR-659, miR-636, miR-638, miR-659, miR-675, miR-1303, miR-1308, miR-1908, miR-1914, miR-1915, miR-2861, miR-3184, miR-4292 and miR-4259 [67]. This group of miRNAs was also found to downregulate the signalling hub which involves Beclin-1 by inhibiting the MAPK/JNK1 signalling pathway [67]. Another five miRNAs were reported to inhibit the ULK kinase complex and these include miR-20a and miR-20b [72], miR-25 [68], miR-489 [37] and miR-1275 [69]. On the other hand, Beclin-1 expression was found to be negatively correlated to the expression of miR-20a [74], miR-21 [75] and miR-221 [76]. MiR-20a could also suppress ATG16L1 expression [74] while miR-567 and miR-638 could negatively regulate ATG5 expression [78, 79]. Overexpression of miR-101 was linked to ATG4 downregulation [80] whereas upregulation of miR-1275 was related to ATG7 suppression [69], and both ATG4 and ATG7 are important in the LC3-II production [16]. Other miRNAs which were involved in suppressing cellular LC3-II production include miR-21 [75], miR-27a [82] and miR-96-5p [83] while miR-638 was found to be involved in promoting LC3-II production [81]. Four miRNAs were reported to be involved in suppressing SQSTM1/p62 expression and these miRNAs include miR-20a [74], miR-27a [82], miR-96-5p [83] and miR-638 [81]

it was also demonstrated to reduce breast cancer resistance towards doxorubicin in vitro and in vivo [37]. In most of the mentioned studies [37, 67, 69], it was shown that autophagy blockage by the corresponding miRNAs would lead to decreased breast cancer tumorigenesis. However, in the combined in vivo and in vitro study that involved miR-25 [68], it was shown that the introduction of miR-25 mimics would restore cellular proliferation and increase cancer cells resistance towards epirubicin, apart from abrogating autophagy. The opposite effects of autophagy on the breast cancer tumorigenesis suggested that autophagy could play the role of a double-edged sword in promoting cancer progression or suppression [70]. FIP200 is another important component of the ULK kinase complex [71], and this protein was found to be negatively regulated by miR-20a and miR-20b [72]. As a result, suppression of FIP200 by these two miRNAs led to autophagy suppression by blocking autophagy initiation step and this also inhibited cancer progression in vitro [72].

Beclin-1 protein is a key component of the class III PI3K complex which plays an essential role in the autophagy initiation step [16]. Bcl-2 is an anti-apoptotic protein that could bind to Beclin-1 to inactivate it and phosphorylation of $\mathrm{Bcl}-2$ by MAPK/JNK1 would dissociate it from Beclin-1, allowing Beclin-1 to initiate the autophagy signalling activity [73]. Same like the case of ULK1 which has been discussed previously, the in vitro study by Ruiz EsparzaGarrido et al. reported that about 18 miRNAs were found to be able to suppress the signaling hub involving MAPK/ JNL1 [67], and thus, this would allow the dephosphorylated $\mathrm{Bcl}-2$ to bind to Beclin-1 to inactivate its activity [73]. Besides, three other miRNAs were shown to negatively regulate Beclin-1 and this include miR-20a [74], miR-21 [75] and miR-221 [76]. Compared to the previous single study that reported 18 potential miRNAs which would suppress 
Table 2 Roles of miRNAs in influencing treatment response in breast cancer by regulating autophagy activities $(n=11)$

\begin{tabular}{|c|c|c|c|c|c|}
\hline Treatment types & Influence on treatment response & miRNA(s) & $\begin{array}{l}\text { Autophagy } \\
\text { activities } \\
(\boldsymbol{\uparrow} / \downarrow)\end{array}$ & Proposed mechanisms/evidences & References \\
\hline \multirow[t]{4}{*}{ Chemotherapy } & Resistant-promoting & $\operatorname{miR}-25$ & $\downarrow$ & $\begin{array}{l}\text { miR-25 downregulated ULK1 to sup- } \\
\text { press autophagy and promote epiru- } \\
\text { bicin resistance in breast cancer cells }\end{array}$ & {$[68]$} \\
\hline & \multirow[t]{3}{*}{ Sensitivity-promoting } & miR-27a & $\downarrow$ & $\begin{array}{l}\text { miR-27a suppressed LC3-II and p62 } \\
\text { expressions to inhibit autophagy and } \\
\text { sensitized cancer cells to doxorubicin } \\
\text { and paclitaxel }\end{array}$ & {$[82]$} \\
\hline & & miR-129-5p & $\downarrow$ & $\begin{array}{l}\text { miR-129-5p suppressed HMGB1 to } \\
\text { inhibit autophagy and sensitized } \\
\text { breast cancer cells to taxol treatment }\end{array}$ & {$[61]$} \\
\hline & & miR-489 & $\downarrow$ & $\begin{array}{l}\text { miR-489 downregulated ULK1 to block } \\
\text { autophagy and sensitized breast can- } \\
\text { cer cells to doxorubicin treatment }\end{array}$ & {$[37]$} \\
\hline \multirow[t]{3}{*}{ Endocrine therapy } & \multirow[t]{2}{*}{ Resistant-promoting } & $\operatorname{miR}-23 b-3 p$ & $\uparrow$ & $\begin{array}{l}\text { miR-23b-3p downregulated SLC6A14 } \\
\text { to promote autophagy and tamoxifen } \\
\text { resistance }\end{array}$ & [75] \\
\hline & & miR-21 & $\downarrow$ & $\begin{array}{l}\text { miR-21 could promote PI3K/AKT/mTOR } \\
\text { pathway to inhibit autophagy and } \\
\text { promote resistance towards both } \\
\text { tamoxifen and fulvestrant }\end{array}$ & [35] \\
\hline & Sensitivity-promoting & miR-101 & $\downarrow$ & $\begin{array}{l}\text { miR-101 suppressed ATG4D, STMN1 } \\
\text { and RAB5A expressions to block } \\
\text { autophagy and promote tamoxifen- } \\
\text { induced cells death }\end{array}$ & {$[80]$} \\
\hline Endocrine and chemotherapy & Sensitivity-promoting & miR-125b-5p & $\uparrow$ & $\begin{array}{l}\text { miR-125b-5p suppressed PAD2 expres- } \\
\text { sion to promote both autophagy and } \\
\text { apoptosis. This sensitized cancer cells } \\
\text { to both tamoxifen and docetaxel }\end{array}$ & [66] \\
\hline \multirow[t]{2}{*}{ Radiotherapy } & \multirow[t]{2}{*}{ Sensitivity-promoting } & miR-26b & $\downarrow$ & $\begin{array}{l}\text { miR-26b downregulated DRAM1 expres- } \\
\text { sion to inhibit autophagy and sensitize } \\
\text { breast cancer cells to irradiation }\end{array}$ & [92] \\
\hline & & miR-200c & $\downarrow$ & $\begin{array}{l}\text { miR-200 downregulated UBQLN1 to } \\
\text { block autophagy and sensitized breast } \\
\text { cancer cells to irradiation }\end{array}$ & [93] \\
\hline Targeted therapy & Sensitivity-promoting & miR-567 & $\downarrow$ & $\begin{array}{l}\text { miR-567 suppressed autophagy by } \\
\text { downregulating ATG5 to sensitize can- } \\
\text { cer cells to trastuzumab treatment }\end{array}$ & [78] \\
\hline
\end{tabular}

11 miRNAs were reported to play essential roles in regulating treatment responses in breast cancer by modulating the cellular autophagy activities [35, $37,93,61$, $66,68,75,78,80,82,92]$. The treatment responses which could be modulated by these autophagy-regulating miRNAs include responses towards chemotherapy [37, $61,66,68,82]$, endocrine therapy [35, 66, 75, 80], radiotherapy [92, 93] and targeted therapy [78]. Out of these 11 miRNAs, only two miRNAs, namely, miR-23b-3p [75] and miR-125b-5p [66], were shown to upregulate autophagy activities while the rest were demonstrated to inhibit autophagy [35, 37, 61, 68, 78, 80, 82, 92, 93]. Upregulation; $\downarrow$ Downregulation

Beclin-1 activity, autophagy and tumorigenicity, these three studies reported enhanced tumerigenicity despite causing autophagy inhibition [74-76]. This suggested that degree of autophagy might influence the survival of cancer cells and excessive autophagy might in fact reduce tumerigenicity [77]. Another possible reason to explain the observance of increase tumerigenicity despite autophagy suppression could be found in one of the studies [75], in which the study discovered that apart from suppressing Beclin-1, miR-21 would also downregulate PTEN to enhance PI3K/Akt signaling pathway. Increase activity of the PI3K/Akt pathway eventually increased tumerigenicity and caused increase resistance of the breast cancer cells towards tamoxifen and fulvestrant in vitro [75]. Therefore, the overall effect on whether an autophagy-regulating miRNA would increase or decrease tumerigenicity also depends on the downstream target or signaling cascade in which it could have exerting effect.

Roles of miRNAs in modulating elongation and nucleation steps during autophagy development by regulating the expression of autophagy-related proteins (ATGs) Another group of key proteins which play vital roles in modulating autophagy process is the autophagy-related 
proteins (ATGs) [49]. A number of miRNAs have been reported to target various ATGs (Fig. 3) and these include miR-20a (ATG16L1) [74], miR-567 and miR-638 (ATG5) [78, 79], miR-101 (ATG4) [80] and miR-1275 (ATG7) [69]. ATG16L1 has been proven to be able to bind to WIPI2 protein directly and this helps recruiting the ATG12/ATG15/ATG16L1 protein complex to the pre-autophagosome (PAS) [46]. MiR-21a was found to downregulate ATG16L1 expression and this suppressed autophagy and increased breast cancer carcinogenesis [74]. ATG5, on the other hand, could be negatively regulated by miR-567 [78] and miR-638 [79], and this resulted in autophagy inhibition. In the study which reported the autophagy inhibitory role of miR-567, it was shown that the breast cancer cells showed increased sensitivity towards trastuzumab treatment in vitro following upregulation of miR-567 and thus, this miRNA is said to suppress both autophagy and breast cancer tumerigenicity [78]. However, in the latter case-control study that involved miR-638, it was shown that ATG5 could be negatively regulated by miR-638, but, low miR-638 expression was associated with poor disease prognosis and enhanced disease progression [79]. This suggested that autophagy suppression by miR-638 was linked to increased cancer tumerigenicity [79], and a more detailed mechanistic study is needed to validate this case-control study finding. ATG4 is a cysteine protein which is involved in converting LC3 to LC3-I before LC3-II can be produced [39]. Overexpression of miR-101 would downregulate ATG4 expression, and, this would lead to autophagy and tumerigenicity suppression [80]. In addition, increased expression of miR-101 would also increase the sensitivity of breast cancer cell line, MCF-7, towards tamoxifen treatment, evidenced by increased cells death [80]. Another miRNA that was shown to involve in regulating ATG expression is miR-1275 in which this miRNA has been demonstrated to suppress ATG7 expression, and this resulted in both autophagy and tumerigenicity suppression [69]. ATG7 has been shown to be involved in converting LC3-I to LC3-II by facilitating the covalent binding of LC3-I with phosphatidylethanolamine at the PAS membrane [39]. Therefore, the suppression of ATG7 would affect the LC3-II production and this would affect the formation of mature autophagosome [49].

\section{Roles of miRNAs in regulating mature autophagosome formation}

On the other hand, several miRNAs were reported to directly regulate the cellular level of LC3-II and miR638 was shown to directly increase the expression of LC3-II in vitro to increase cellular autophagy and tumorigenesis [81]. This study finding contradicts another study finding which stated that miR-638 was involved in downregulating autophagy by targeting ATG5 and ATG-2B [79]. In another study [67], upregulation of miR638 was found to enhance cellular LC3-II level, but, the authors described that this phenomenon could be related to other stimulus induction as most of the autophagyrelated transcripts were downregulated when miR-638 expression was increased. Therefore, more functional assay may need to be conducted to clarify the exact role of miR-638 in regulating cellular autophagy and tumorigenesis. For miRNAs that were shown to correlate to decreased cellular LC3-II level, three miRNAs were reported and these include miR-21 [75], miR-27a [82] and miR-96-5p [83]. For miR-21, apart from being demonstrated to decrease cellular LC3-II level, miR21 was also shown to suppress Beclin-1 expression and these combined effects would inhibit autophagy in vitro [75]. The upregulation of both miR-27a and miR-96-5p were proven to reduce cellular level of LC3-II to suppress autophagy and tumorigenesis $[82,83]$. In addition, overexpression of miR-27a was also shown to improve the breast cancer cells sensitivity towards doxorubicin and paclitaxel in vitro [82]. Besides, both miR-27a and miR-96-5p were proven to reduce the cellular level of SQSTM1 protein in vitro $[82,83]$. SQSTM1 is an important ubiquitination protein that tags the macromolecules or cargo to be degraded so that these substances can be recognized and bind to the autophagy initiating complex [18]. Downregulation of SQSTM1 would impair substrates delivery to the autophagosome and this would results in the accumulation of aggregated protein and thus, this would eventually lead to inflammatory and degenerative disease [18]. Other miRNA which was shown to suppress cellular SQSTM1 includes miR-20a and this miRNA also downregulates other autophagy related proteins like ATG16L1 and Beclin 1 [74].

Even though multiple miRNAs have been reported to play essential roles in regulating autophagy initiation, elongation, nucleation and autophagosome formation, however, to the best of our knowledge, there is a lack of study which has reported any miRNA that could target the key proteins (LAMP2, SNARE, Rab GTPase RAB7) which are important in regulating the fusion of autophagosome and lysosome to form autolysosome.

\section{Roles of miRNAs in regulating autophagy by modulating expressions of other proteins that do not involve directly in autophagy development}

Apart from regulating the key proteins involved in the autophagy signaling pathway (Fig. 3), there are also some miRNAs which could modulate the autophagy activities by regulating other cellular proteins which are not involved directly in the autophay signaling pathway. SLC6A14 is a type of basic amino acid transporter that 
is negatively regulated by miR-23b-3p and the downregulation of this transporter would result in the increased influx of acidic amino acids via another transporter, SLC1A2 [35]. The disruption in the cellular amino acid level may promote autophagy [84] and SLC6A14 blockage has been shown to promote autophagy in colon cancer [85]. As a result, miR-23b-3p was shown to enhance both autophagy and tumorigenesis, and increase resistance of the breast cancer cells towards hormonal drugs like fulvestrant and tamoxifen [35]. MiR-126 was reported to downregulate the IRS/Glut-4 signaling pathway and this would cause cellular energy deprivation [86]. It has been widely established that glucose depletion is an essential factor that triggers autophagy [87] and thus, miR-126 is said to induce autophagy by activating the AMPK/autophagy signaling cascade [86]. PAD2 is a corepressor of tumour suppressor p53 protein [88] and p53 can negatively regulate PI3K/Akt signaling pathway [89]. MiR-125b-5p was reported to downregulate PAD2 and this would increase p53 expression that inhibits PI3K/Akt signaling [66]. Blockage of PI3K/Akt signaling pathway would enhance autophagy as mTOR is downregulated and the net effect was increased autophagy, decreased cancer cells proliferation and increased cancer cells sensitivity towards tamoxifen and docetaxel [66]. HMGB1 is an autophagy-regulating protein that functions to displace Bcl-2 to bind to Beclin-1, in order to activate Beclin-1 for autophagy induction [90]. An in vitro study involving MCF-7 cancer cell line showed that overexpression of miR-129-5p would downregulate HMGB1 and therefore, this suppressed both autophagy and tumorigenesis [61]. In addition, upregulation of miR129-5p was also found to improve MCF-7 sensitivity towards taxol in vitro [61]. Another miRNA which was reported to downregulate HMGB1 is miR-107 [36] and like miR-129-5p, miR-107 suppressed both autophagy and cancer cells proliferation by downregulating HMGB1 expression.

DRAM1 is identified as a direct downstream target of p53 and its detailed role in autophagy modulation is still not fully understood [91]. DRAM1 may be involved in increasing lysosomal acidification or inhibiting Akt signaling pathway to promote cellular autophagy [91]. In a combined clinical and in vitro study [92], it was shown that overexpression of miR26b would negatively regulate DRAM1 expression and this resulted in decreased autophagy, tumorigenesis and improved cancer cells sensitivity towards radiotherapy. In another combined clinical and in vitro study [93], ectopic expression of miR-200c was shown to downregulate UBQLN1 to suppress autophagy and tumorigenesis, and sensitized the breast cancer cells towards radiotherapy. UBQLN proteins like UBQLN1 and UBQLN4 have been reported to play some roles during the formation of autophagosome and these proteins might also help in the fusion of autophagosome and lysosome to form autolysosome [94]. XIAP is an anti-apoptotic protein in which its role in autophagy modulation remains controversy [95]. MiR-23a was reported to downregulate XIAP to increase autophagy and tumorigenesis in a published study finding [96], however, the exact molecular mechanism on how XIAP dowregulation led to enhanced autophagy was not explored further. One possible explanation is that XIAP might play certain role in inhibiting MDM2/p53 signaling pathway to block autophagy induction [95] and by suppressing XIAP expression, autophagy could be induced [96].

Fundc1 is an important mammalian mitochondrion membrane protein which plays an essential role in recruiting LC3 to the mitochondrion to initiate mitophagy [97]. It has been reported that Fundc1 could also upregulate the expression of autophagyrelated proteins like Beclin-1, ATG5 and ATG7, and apoptotic-related proteins like BAX [98]. In view of the multiple autophagy roles that could be played by Fundc1 protein, a group of researchers investigated the potential role of miR-137 in modulating Fundc1 expression and they found that this protein could be negatively regulated by miR-137 [99]. Downregulation of Fundc1 following miR-137 overexpression would subsequently promote tumorigenesis [99] and the reason of increased tumorigenesis could be related to the downregulation of the apoptotic-related genes following Fundc1 downregulation [98]. Another protein which has been reported to be involved in regulating the transcription of autophagy-related genes is Smad4 and it is part of the TGF- $\beta$ regulated signaling pathway [100]. The activation of the TGF- $\beta / \operatorname{Smad} 4$ signaling pathway will increase the expression of several key autophagy-related proteins like ATG5, ATG6 and ATG7 [100]. MiR-224-5p was reported to suppress Smad4 expression and autophagy in vitro and this resulted in increased cancer cells tumerigenicity [101]. Like Fundc1, Smad4 also involves in upregulating expression of apoptotic-related proteins like $\mathrm{Bcl}, \mathrm{BIK}$ and BIM [100] and thus, suppression of Smad4 would lead to apoptosis inhibition and cause increase in the cancer progression [101]. Let-7a, on the other hand, was reported to downregulate both autophagy and cancer cells tumerigenicity in vitro but its direct downstream target was unreported [102]. However, let-7a has been reported to promote autophagy in other solid cancers like gastric cancer [26] and lung cancer [29]. Therefore, 
more study is needed to confirm whether let-7a would suppress autophagy in human breast cancer cells.

\section{Roles of autophagy-regulating miRNAs in modulating treatment response in breast cancer}

From all the previously discussed autophagy-regulating miRNAs, 11 miRNAs were further reported to be involved in regulating treatment responses towards chemotherapy [37, 61, 66, 68, 82], endocrine therapy $[35,66,75,80]$, radiotherapy [92, 93] and targeted therapy [78] in breast cancer (Table 2). Four miRNAs, namely, miR-25, miR-27a, miR-129-5p and miR-489 were reported to suppress autophagy in breast cancer cells [37, $61,68,82]$. However, miR-25 was the only miRNA which was demonstrated to promote chemoresistant in breast cancer [68] while the other three miRNAs were proven to promote chemosensitivity in breast cancer [37, 61, 82]. As autophagy has been reported to be able to exert both tumour-promoting and tumour-suppressing effects [61], it is therefore not surprising that autophagy induction or inhibition would promote treatment resistance in some study while in other study, autophagy dysregulation could promote sensitivity towards a specific cancer therapy. Similar phenomenon was observed when it was shown that both miR-21 and miR-101 would suppress cellular autophagy activities but the former miRNA would promote resistance towards endocrine therapy [35] while the latter one would promote sensitivity towards endocrine therapy [80]. Compared to miR-21 and miR-101, miR23b-3p was the only miRNA which was shown to promote both autophagy and resistance towards endocrine therapy in breast cancer [75]. Other than miRNA, other non-coding RNAs like long non-coding RNA H19 has also been reported to be able to promote both autophagy and tamoxifen resistance in breast cancer [103]. On the other hand, miR-125b-5p was recognized as the autophagy-promoting miRNA that could induce sensitivity towards both chemotherapy and hormonal therapy by upregulating cellular autophagy and apoptosis [66]. This suggests that miR-125b-5p could be possibly further studied to investigate its potential to be used in clinical trial to improve sensitivity of the breast cancer patients towards both chemotherapy and endocrine therapy.

For radiotherapy, miR-26b and miR-200c were reported to be autophagy-inhibiting miRNAs that promote radiosensitivity [92, 93]. These findings probably suggested that autophagy could be important in providing nutrients for the cancer cells to maintain cellular homeostasis during radiotherapy [63]. Similarly, miR-567 was also shown to suppress autophagy and promote sensitivity towards targeted therapy in breast cancer [78]. Again, this further supported the essential role of autophagy in ensuring the cancer cells survival when targeted therapy is administered [64]. One important point to take note of is that it can be observed that autophagy induction or inhibition in chemotherapy and endocrine therapy could either promote treatment resistance or treatment sensitivity but in both radiotherapy and targeted therapy, autophagy inhibition seems to promote treatment sensitivity only than treatment resistance.

\section{Autophagy-regulating miRNAs as potential breast cancer biomarkers and therapeutic agents: applications, challenges and recommendations}

As discussed in the previous section, it can be clearly seen that a number of miRNAs (Table 1) has been demonstrated to influence the breast cancer progression or treatment response by modulating the cellular autophagy process. By understanding the relationships between a specific miRNA and its effect on the cellular autophagy modulation and tumorigenesis, it helps enabling this miRNA to be employed as the breast cancer biomarker [37, 79]. Take miR-125b-5p as an example, upregulation of this miRNA would suppress breast cancer tumorigenesis and sensitize the breast cancer cells towards tamoxifen and docetaxel by accelerating both apoptosis and autophagy [66]. The autophagy enhancement was achieved by downregulating PAD2 protein expression [66] and this protein is a corepressor for tumour suppressor protein $\mathrm{p} 53$ [88]. Therefore, by monitoring the level of miR-125b-5p in the human breast cancer patients, it may help to monitor the disease progression and predict the response towards hormonal and chemotherapy. Besides, miRNA can also be utilized as a novel therapeutic agent to tackle breast cancer progression [35, 99]. For instance, miR-107 has been proven to inhibit breast cancer cells proliferation, migration and autophagy in vivo and in vitro by targeting HMGB1 [36]. This generates a possibly to increase the expression of miR-107 level in the human breast cancer patients to slow breast cancer progression [36]. In short, the use of miRNAs as cancer biomarkers and therapeutic agents may help allow disease detection and progress monitoring, which will then help the patients to improve their treatment response and survival [104].

Autophagy can be a double-edged sword in either promoting cancer progression or suppression [61, 70]. Autophagy may help generating nutrients for the cancer cells to grow and remove dysfunctional cellular macromolecules but excessive autophagy may lead to cellular death $[55,77]$. Even though numerous studies have proposed the potential roles of autophagy-regulating miRNAs as cancer biomarkers or therapeutic agents, one problem faced is that some autophagy-regulating miRNA seems to exert different effects on the breast cancer tumorigenesis in different studies. By taking miR-20a as an 
example, in the study reported by Liu et al., it was shown that miR-20a would suppress autophagy but enhance breast cancer progression in vivo and in vitro [74]. However, in another study, it was demonstrated that miR20a would suppress both autophagy and breast cancer progression [72]. Both studies reported autophagy suppression by miR-20a but this miRNA would exert different effects on the breast cancer tumorigenesis in two different studies [72, 74]. Therefore, it is inconclusive to say whether miR-20a upregulation in the breast cancer patient is a good or bad sign and thus, more further study is needed to validate the exact role of miR-20a clinically, before this miRNA can be used as a breast cancer biomarker or therapeutic agent.

Another problem with the potential use of miRNAs as cancer biomarker is that it requires many detailed and independent testings, before a panel of effective and specific cancer biomarkers can be introduced [105]. To the best of our knowledge, currently there is no a panel of autophagy-regulating miRNAs which has gone through multiple testings to prove its effectiveness and specificity. So, it is suggested that future study can focus on the list of autophagy-regulating miRNAs which have been reported to play roles in human breast cancer development, and from the list, more detailed study can be conducted to evaluate the suitability of these miRNAs as breast cancer biomarkers. As for the use of miRNA as potential cancer therapeutic agent, several problems like suitable delivery methods and unwanted off-target effects are still remain unsolved [106], and thus, there is still a long way to go before autophagy-regulating miRNAs can be certified safe to be employed as the novel breast cancer therapeutic agent.

\section{Conclusions}

This review effectively summarizes the findings from various in vitro, in vivo and clinical studies on the roles of a number of autophagy-regulating miRNAs in influencing the human breast cancer progression and treatment response. By modulating the cellular autophay process, these miRNAs could actually suppress or enhance the breast cancer progression. Therefore, these miRNAs have great potentials to be developed into useful breast cancer biomarkers or new therapeutic agent. To make this happens, more detailed mechanistic and clinical trials should be conducted to evaluate the safety, specificity, sensitivity and effectiveness of these miRNAs as breast cancer biomarkers and therapeutic agents.

\footnotetext{
Abbreviations

AMBRA: Autophagy and beclin-1 regulator; AMPK: Adenosine monophosphate-activated protein kinase; ATG16L1: Autophagy related 16 like 1; ATG4D: Autophagy related 4D cysteine peptidase; BAX: Bcl-2-associated X
}

protein; BCl-2: B cell lymphoma 2; BECN1: Beclin-1; Bif-1: Endophilin; BIK: BCl-2 interacting killer protein; BIM: BCl-2 like protein 11; CMA: Chaperon-mediated autophagy; DACT3: Dishevelled binding antagonist of beta catenin 3; DFCP1: Zinc-finger FYVE domain-containing protein 1; DRAM1: DNA damageregulated autophagy modulator protein 1; ER: Endoplasmic reticulum; ESCRT/ III: Endosomal sorting complexes required for transport I and III; FIP200: FAK family kinase-interacting protein of 200 kDa; FOXO1: Forkhead box protein 01; Fundc1: FUN14 domain-containing protein 1; FYCO1: FYVE and coiledcoil domain-containing protein 1; GFAP: Glial fibrillary acidic protein; Glut-4: Glucose transporter type 4; HGS: Hepatocyte growth factor-regulated tyrosine kinase substrate; HMGB1: High mobility group box 1 protein; HOPS: Homotypic fusion and protein sorting protein complex; HSC70: Heat shock cognate 71 kDa protein; HSP90: Heat shock protein 90; IRS: Insulin receptor substrate; JNK1: c-Jun N-terminal kinase 1; LAMP-A2: Lysosome-associated membrane protein type 2a; LAPTM4B: Lysosomal protein transmembrane 4 beta; LC3-I/II: LC3-phosphatidylethanolamine conjugate I/II; LKB1: Serine/threonine-protein kinase STK11; LYNUS: Lysosome nutrient sensing machinery; MAPK: Mitogen activated protein kinase; MDM2: Mouse double minute 2 homolog; MiRNA: MicroRNA; mRNA: Messenger RNA; mTOR: Mammalian target of rapamycin; NBR1: Next to BRCA1 gene 1 protein; ncRNA: Non-coding RNA; NBR1: Next to BRCA1 gene 1 protein; NOD: Non-obese diabetic; PAD2: Peptidylarginine deiminase 2; PAS: Pre-autophagosome; PE: Phosphatidylethanolamine; PI3K: Phosphoinositide 3-kinase; PIP2: Phosphatidylinositol-4,5-bisphosphate; PIP3: Phosphatidylinositol-3,4,5-bisphosphate; PKB/Akt: Protein kinase B; PLEKHM1/2: Pleckstrin homology domain-containing family M member 1/2; PML: Promyelocytic leukemia protein; PTEN: Phosphatase and tensin homolog; RAB5A: Ras-related protein Rab-5A; RBCC1: RB1-inducible coiled-coil protein 1; RILP: Rab-interacting lysosomal protein; RNA: Ribonucleic acid; RTK: Receptor tyrosine kinase; SCID: Severe combined immunodeficiency disease; SLC6A 14: Sodium- and chloride-dependent neutral and basic amino acid transporter B; Smad4: SMAD family member 4; SNARE: Soluble NSF attachment protein receptor; SNCA: a-Synuclein; SQSTM1: Sequestosome-1; STMN1: Stathmin 1; TECPR1: Tectonin beta-propeller repeat containing 1; TFEB: Transcription factor EB; TGF- $\beta$ : Transforming growth factor $\beta$; TSC $1 / 2$ : Tuberous sclerosis 1/2; UBQLN1: Ubiquilin-1; ULK1: Unc-51 like autophagy activating kinase; VPS15/34: Vacuolar protein sorting15/34; WIPI2: WD repeat domain phosphoinositide-interacting protein $2 ;$ XIAP: X-linked inhibitor of apoptosis protein; 3'-UTR: 3'-Untranslated region; 4-OHT: 4-hydroxytamoxifen.

\section{Acknowledgements}

Zhi Xiong Chong received scholarship from University of Nottingham Malaysia. Authors wish to thanks MOHE, Malaysia for funding this study.

\section{Authors' contributions}

WYH and SKY conceptualized the idea, developed the structure of the article and guided the selection of references; ZXC was the major contributor in writing the manuscript; ZXC prepared the figures/Tables. All authors read and approved the final manuscript.

Funding

This work was supported by FRGS grant by Ministry of Higher Education, Malaysia, Grant number FRGS/1/2018/STG05/UNIM/02/1 and FRGS/1/2014/ SG05/UNIM/02/1.

\section{Availability of supporting data}

All data was included in the manuscript.

\section{Declarations}

Ethics approval and consent to participate Not applicable.

\section{Consent for publication}

Not applicable.

\section{Competing interests}

The authors report no conflict of interest. 


\begin{abstract}
Author details
${ }^{1}$ Faculty of Science and Engineering, University of Nottingham Malaysia, 43500 Semenyih, Selangor, Malaysia. ${ }^{2}$ China-ASEAN College of Marine Sciences, Xiamen University Malaysia, 43900 Sepang, Selangor, Malaysia.
\end{abstract}

Received: 2 December 2020 Accepted: 25 February 2021 Published online: 25 March 2021

\section{References}

1. World Health Organizations. Human breast cancer. 2020. https://www who.int/cancer/prevention/diagnosis-screening/breast-cancer/en/. Accessed 8 Mar 2020.

2. American Cancer Society. Breast cancer facts and figs. 2019-2020. Am Cancer Soc. 2020. https://www.cancer.org/content/dam/cancer-org/ research/cancer-facts-and-statistics/breast-cancer-facts-and-figures/ breast-cancer-facts-and-figures-2019-2020.pdf. Accessed 24 Oct 2020

3. Januškevičienè I, Petrikaitè V. Heterogeneity of breast cancer: The importance of interaction between different tumor cell populations. Life Sci. 2019;239:117009. https://doi.org/10.1016/j.lfs.2019.117009.

4. Ji X, Lu Y, Tian H, Meng X, Wei M, Cho WC. Chemoresistance mechanisms of breast cancer and their countermeasures. Biomed Pharmacother. 2019:114:108800.

5. Gray M, Turnbull AK, Ward C, Meehan J, Martínez-Pérez C, Bonello M, et al. Development and characterisation of acquired radioresistant breast cancer cell lines. Radiat Oncol. 2019;14:64

6. Fan W, Chang J, Fu P. Endocrine therapy resistance in breast cancer: current status, possible mechanisms and overcoming strategies. Future Med Chem. 2015;7:1511-9.

7. Derakhshani A, Rezaei Z, Safarpour H, Sabri M, Mir A, Sanati MA, et al. Overcoming trastuzumab resistance in HER2-positive breast cancer using combination therapy. J Cell Physiol. 2020;235:3142-56.

8. Yu Y, Cao XC. MiR-190-5p in human diseases. Cancer Cell Int. 2019:19:257. https://doi.org/10.1186/s12935-019-0984-X.

9. Loh H-Y, Norman BP, Lai K-S, Rahman NMANA, Alitheen NBM, Osman MA. The regulatory role of microRNAs in breast cancer. Int J Mol Sci. 2019;20:4940

10. Brien JO, Hayder H, Zayed $Y$, Peng C. Overview of microRNA biogenesis, mechanisms of actions, and circulation. Front Endocrinol (Lausanne). 2018;9:402

11. Mayya VK, Duchaine TF. Ciphers and executioners: how 3 primeuntranslated regions determine the fate of messenger RNAs. Front Genet. 2019;10:6.

12. Hamam R, Hamam D, Alsaleh KA, Kassem M, Zaher W, Alfayez M, et al. Circulating microRNAs in breast cancer: novel diagnostic and prognostic biomarkers. Cell Death Dis. 2017;8:e3045. https://doi.org/10.1038/ cddis.2017.440

13. Rehman $\mathrm{O}$, Zhuang $\mathrm{H}$, Ali AM, Ibrahim A, Li Z. Validation of miRNAs as breast cancer biomarkers with a machine learning approach. Cancers (Basel). 2019:11:431

14. Mehrgou A, Akouchekian M. Therapeutic impacts of microRNAs in breast cancer by their roles in regulating processes involved in this disease. J Res Med Sci. 2017;22:130.

15. Hanna J, Hossain GS, Kocerha J. The potential for microRNA therapeutics and clinical research. Front Genet. 2019;10:478.

16. Khandia R, Dadar M, Munjal A, Tiwari KDKKR, Yatoo MI, lqbal HMN, et al. A comprehensive review of autophagy and its various roles in infectious, non-infectious, and lifestyle diseases: current knowledge and prospects for disease prevention, novel drug design, and therapy. Cells. 2019;8:674

17. Flynn ALB, Schiemann WP. Autophagy in breast cancer metastatic dormancy: tumor suppressing or tumor promoting functions? J Cancer Metastasis Treat. 2019:5:43.

18. Kirkin V. History of the selective autophagy research: how did it begin and where does it stand today? J Mol Biol. 2020;432:3-27. https://doi. org/10.1016/j.jmb.2019.05.010.

19. Kim YS, Silwal P, Kim SY, Yoshimori T, Jo EK. Autophagy-activating strategies to promote innate defense against mycobacteria. Exp Mol Med. 2019;51:151. https://doi.org/10.1038/s12276-019-0290-7.
20. Xiong H, Shen J, Chen Z, Yang J, Xie B, Jia Y, et al. H19/let-7/Lin28 ceRNA network mediates autophagy inhibiting epithelial-mesenchymal transition in breast cancer. Int J Oncol. 2020;56:794-806.

21. Devenport SN, Shah YM. Functions and implications of autophagy in colon cancer. Cells. 2019:8:1349.

22. Abdellatif M, Sedej S, Carmona-Gutierrez D, Madeo F, Kroemer G. Autophagy in cardiovascular aging. Circ Res. 2018;123:803-24.

23. Chen H-X, Liang F-C, Gu P, Xu B-L, Xu H-J, Wang W-T, et al. Exosomes derived from mesenchymal stem cells repair a Parkinson's disease model by inducing autophagy. Cell Death Dis. 2020;11:288. https://doi. org/10.1038/s41419-020-2473-5.

24. Uddin MS, Stachowiak A, Al Mamun A, Tzvetkov NT, Takeda S, Atanasov $A G$, et al. Autophagy and Alzheimer's disease: from molecular mechanisms to therapeutic implications. Front Aging Neurosci. 2018;10:04.

25. Guo Y, Zhang X, Wu T, Hu X, Su J, Chen X. Autophagy in skin diseases. Dermatology. 2019;235:380-9.

26. Fan H, Jiang M, Li B, He Y, Huang C, Luo D, et al. MicroRNA-let-7a regulates cell autophagy by targeting Rictor in gastric cancer cell lines MGC-803 and SGC-7901. Oncol Rep. 2018;39:1207-14.

27. Gu J, Wang Y, Wang $X$, Zhou D, Wang X, Zhou M, et al. Effect of the IncRNA GAS5-miR-23a-ATG3 axis in regulating autophagy in patients with breast cancer. Cell Physiol Biochem. 2018;48:194-207.

28. Xu S, Wang P, Zhang J, Wu H, Sui S, Zhang J, et al. Ai-IncRNA EGOT enhancing autophagy sensitizes paclitaxel cytotoxicity via upregulation of ITPR1 expression by RNA-RNA and RNA-protein interactions in human cancer. Mol Cancer. 2019;18:89.

29. Duan S, Yu S, Yuan T, Yao S, Zhang L. Exogenous let-7a-5p induces A549 lung cancer cell death through BCL2L1-mediated PI3Ky signaling pathway. Front Oncol. 2019;9:808.

30. Djavaheri-Mergny M, Giuriato S, Tschan MP, Humbert M. Therapeutic modulation of autophagy in leukaemia and lymphoma. Cells. 2019:8:103.

31. Gan X, Zhu H, Jiang X, Obiegbusi SC, Yong M, Long X, et al. CircMUC16 promotes autophagy of epithelial ovarian cancer via interaction with ATG13 and miR-199a. Mol Cancer. 2020;19:45.

32. Fazio P, Di Matrood S. Targeting autophagy in liver cancer. Transl Gastroenterol Hepatol. 2018;3:39.

33. Amaravadi RK, Kimmelman AC, Debnath J. Targeting autophagy in cancer: recent advances and future directions. Cancer Discov. 2019:9:1167-81.

34. Yun CW, Lee SH. The roles of autophagy in cancer. Int J Mol Sci. 2018:19:3466

35. Bacci M, Lorito N, Ippolito L, Ramazzotti M, Luti S, Romagnoli S, et al. Reprogramming of amino acid transporters to support aspartate and glutamate dependency sustains endocrine resistance in breast cancer. Cell Rep. 2019;28:104-18.e8.

36. Ai H, Zhou W, Wang Z, Qiong G, Chen Z, Deng S. microRNAs-107 inhibited autophagy, proliferation, and migration of breast cancer cells by targeting HMGB1. J Cell Biochem. 2019:120:8696-705.

37. Soni M, Patel Y, Markoutsa E, Jie C, Liu S, Xu P, et al. Autophagy, cell viability and chemo-resistance are regulated by miR-489 in breast cancer. Mol Cancer Res. 2018;16:1348-60.

38. Yao R-Q, Ren C, Xia Z-F, Yao Y-M. Organelle-specific autophagy in inflammatory diseases: a potential therapeutic target underlying the quality control of multiple organelles. Autophagy. 2020;12:1-17. https://doi. org/10.1080/15548627.2020.1725377.

39. Acevo-rodríguez PS, Maldonado G, Castro-obregón S, Hernandez G. Autophagy regulation by the translation machinery and its implications in cancer. Front Oncol. 2020;10:322.

40. Tompkins KD, Thorburn A. Regulation of apoptosis by autophagy to enhance cancer therapy. Yale J Biol Med. 2019;92:707-18.

41. Gozuacik D, Akkoc Y, Gulfem Ozturk D, Kocak M. Autophagy-regulating microRNAs and cancer. Front Oncol. 2017;7:65.

42. Yim WWY, Mizushima N. Lysosome biology in autophagy. Cell Discov. 2020;6:6. https://doi.org/10.1038/s41421-020-0141-7.

43. Dong S, Aguirre-Hernandez C, Scrivo A, Eliscovich C, Arias E, BravoCordero JJ, et al. Monitoring spatiotemporal changes in chaperonemediated autophagy in vivo. Nat Commun. 2020;11:645.

44. Slobodnyuk K, Radic N, Ivanova S, Llado A, Trempolec N, Zorzano A, et al. Autophagy-induced senescence is regulated by p38a signaling Cell Death Dis. 2019:10:376. 
45. Kotani T, Kirisako H, Koizumi M, Ohsumi Y, Nakatogawa H. The Atg2Atg18 complex tethers pre-autophagosomal membranes to the endoplasmic reticulum for autophagosome formation. Proc Natl Acad Sci USA. 2018;115:10363-8.

46. Dikic I, Elazar Z. Mechanism and medical implications of mammalian autophagy. Nat Rev Mol Cell Biol. 2018;19:349-64. https://doi.org/10. 1038/s41580-018-0003-4.

47. Li F, Guo H, Yang Y, Feng M, Liu B, Ren X, et al. Autophagy modulation in bladder cancer development and treatment (Review). Oncol Lett. 2019:42:1647-55

48. Graef M. Recent advances in the understanding of autophagosome biogenesis. F1000 Res. 2020;9:F1000 Faculty Rev-212.

49. Li X, He S, Ma B. Autophagy and autophagy-related proteins in cancer. Mol Cancer. 2020;19:12

50. Agrotis A, Chamier L, Von, Oliver H, Kiso K, Singh T, Ketteler R. Human ATG4 autophagy proteases counteract attachment of ubiquitinlike LC3/GABARAP proteins to other cellular proteins. J Biol Chem. 2019:294:12610-21.

51. Lorincz P, Juhasz G. Autophagosome-lysosome fusion. J Mol Biol. 2020:432:2462-82.

52. Voisin A, Monville C, Plancheron A, Béré E, Gaillard A, Leveziel N. Cathepsin $\mathrm{B} \mathrm{pH}$-dependent activity is involved in lysosomal dysregulation in atrophic age-related macular degeneration. Oxid Med Cell Longev. 2019:2019:5637075.

53. Li J, Balboula AZ, Aboelenain M, Fujii T, Moriyasu S, Bai H, et al. Effect of autophagy induction and cathepsin B inhibition on developmental competence of poor quality bovine oocytes. J Reprod Dev. 2020;66:83-91.

54. Holczer M, Hajdú B, Lorincz T, Szarka A, Bánhegyi G, Kapuy O. A double negative feedback loop between mTORC1 and AMPK kinases guarantees precise autophagy induction upon cellular stress. Int J Mol Sci. 2019;20:5543.

55. Heras-Sandoval D, Pérez-Rojas JM, Hernández-Damián J, PedrazaChaverri J. The role of PI3K/AKT/mTOR pathway in the modulation of autophagy and the clearance of protein aggregates in neurodegeneration. Cell Signal. 2014;26:2694-701. https://doi.org/10.1016/j.cells ig.2014.08.019.

56. Ilisso CP, Delle Cave D, Mosca L, Pagano M, Coppola A, Mele L, et al. S-Adenosylmethionine regulates apoptosis and autophagy in MCF-7 breast cancer cells through the modulation of specific microRNAs. Cancer Cell Int. 2018;18:197. https://doi.org/10.1186/ s12935-018-0697-6.

57. Chen Y, Zhang W, Guo X, Ren J, Gao A. The crosstalk between autophagy and apoptosis was mediated by phosphorylation of $\mathrm{BCl}-2$ and beclin1 in benzene-induced hematotoxicity. Cell Death Dis. 2019;10:772. https://doi.org/10.1038/s41419-019-2004-4.

58. Lee W, Kim SH. Autophagy at synapses in neurodegenerative diseases. Arch Pharm Res. 2019;42:407-15. https://doi.org/10.1007/ s12272-019-01148-7.

59. Nnah IC, Wang B, Saqcena C, Weber GF, Bonder EM, Bagley D, et al. TFEB-driven endocytosis coordinates MTORC1 signaling and autophagy. Autophagy. 2019;15:151-64. https://doi.org/10.1080/ 15548627.2018.1511504.

60. Settembre C, Ballabio A. Lysosomal adaptation: how the lysosome responds to external cues. Cold Spring Harb Perspect Biol. 2014;6:a016907.

61. Shi Y, Gong W, Lu L, Wang Y, Ren J. Upregulation of miR-129-5p increases the sensitivity to taxol through inhibiting HMGB1-mediated cell autophagy in breast cancer MCF-7 cells. Braz J Med Biol Res. 2019;52:e8657.

62. Zarzynska JM. The importance of autophagy regulation in breast cancer development and treatment. Biomed Res Int. 2014;2014:710345.

63. Tam SY, Wu VWC, Law HKW. Influence of autophagy on the efficacy of radiotherapy. Radiat Oncol. 2017;12:57.

64. Mele L, Vecchio V del, Liccardo D, Prisco C, Schwerdtfeger M, Robinson $\mathrm{N}$, et al. The role of autophagy in resistance to targeted therapies. Cancer Treat Rev. 2020;88:102043.

65. Berardi DE, Campodónico PB, Díaz Bessone MI, Urtreger AJ, Todaro LB. Autophagy: friend or foe in breast cancer development, progression, and treatment. Int J Breast Cancer. 2011;2011:595092.
66. Li F, Miao L, Xue T, Qin H, Mondal S, Thompson PR, et al. Inhibiting PAD2 enhances the anti-tumor effect of docetaxel in tamoxifen-resistant breast cancer cells. J Exp Clin Cancer Res. 2019;38:414.

67. Ruiz Esparza-Garrido R, Torres-Márquez ME, Viedma-Rodríguez $R$, Velázquez-Wong AC, Salamanca-Gómez F, Rosas-Vargas H, et al. Breast cancer cell line MDA-MB-231 miRNA profile expression after BIK interference: BIK involvement in autophagy. Tumor Biol. 2016;37:6749-59. https://doi.org/10.1007/s13277-015-4494-8.

68. Wang Z, Wang N, Liu P, Chen Q, Situ H, Xie T, et al. MicroRNA-25 regulates chemoresistance-associated autophagy in breast cancer cells, a process modulated by the natural autophagy inducer isoliquiritigenin. Oncotarget. 2014;5:7013-26.

69. Liang G, Ling Y, Mehrpour M, Saw PE, Liu Z, Tan W, et al. Autophagyassociated circRNA circCDYL augments autophagy and promotes breast cancer progression. Mol Cancer Molecular Cancer. 2020;19:65.

70. Mirza-Aghazadeh-Attari M, Mohammadzadeh A, Adib A, Darband SG, Sadighparvar S, Mihanfar A, et al. Melatonin-mediated regulation of autophagy: making sense of double-edged sword in cancer. J Cell Physiol. 2019;234:17011-22.

71. Tian W, Alsaadi R, Guo Z, Kalinina A, Carrier M, Tremblay M, et al. An antibody for analysis of autophagy induction. Nat Methods. 2020;17:232-9. https://doi.org/10.1038/s41592-019-0661-y.

72. Li S, Qiang Q, Shan H, Shi M, Gan G, Ma F, et al. MiR-20a and miR-20b negatively regulate autophagy by targeting RB1CC1/FIP200 in breast cancer cells. Life Sci. 2016;147:143-52. https://doi.org/10.1016/j.lfs.2016. 01.044.

73. Zhou YY, LiY, Jiang WQ, Zhou LF. MAPK/JNK signalling: a potential autophagy regulation pathway. Biosci Rep. 2015;35:e00199.

74. Liu L, He J, Wei X, Wan G, Lao Y, Xu W, et al. MicroRNA-20a-mediated loss of autophagy contributes to breast tumorigenesis by promoting genomic damage and instability. Oncogene. 2017;36:5874-84.

75. Yu X, Li R, Shi W, Jiang T, Wang Y, Li C, et al. Silencing of MicroRNA-21 confers the sensitivity to tamoxifen and fulvestrant by enhancing autophagic cell death through inhibition of the PI3K-AKT-mTOR pathway in breast cancer cells. Biomed Pharmacother. 2016;77:37-44. https://doi.org/10.1016/j.biopha.2015.11.005.

76. Pradhan AK, Talukdar S, Bhoopathi P, Shen X-N, Emdad L, Das SK, et al. mda-7/L-24 mediates cancer cell-specific death via regulation of miR221 and the beclin-1 axis. Cancer Res. 2017;77:949-59.

77. Sun J, Feng Y, Wang Y, Ji Q, Cai G, Shi L, et al. a-hederin induces autophagic cell death in colorectal cancer cells through reactive oxygen species dependent AMPK/mTOR signaling pathway activation. Int J Oncol. 2019;54:1601-12.

78. Han M, Hu J, Lu P, Cao H, Yu C, Li X, et al. Exosome-transmitted miR-567 reverses trastuzumab resistance by inhibiting ATG5 in breast cancer. Cell Death Dis. 2020;11:43. https://doi.org/10.1038/s41419-020-2250-5.

79. Ahmadi S, Saffari M, Modarressi MH, Shirkoohi R, Jamalian M, Teimori H. Dysregulation of miR-638 in breast cancer patients and bioinformatics investigation of its target genes in apoptosis, angiogenesis and autophagy pathways. Int J Cancer Manag. 2019;12:e88829.

80. Frankel LB, Wen J, Lees M, Høyer-Hansen M, Farkas T, Krogh A, et al. MicroRNA-101 is a potent inhibitor of autophagy. EMBO $\mathrm{J}$. 2011;30:4628-41.

81. Ren Y, Chen Y, Liang X, Lu Y, Pan W, Yang M. MiRNA-638 promotes autophagy and malignant phenotypes of cancer cells via directly suppressing DACT3. Cancer Lett. 2017;390:126-36.

82. Ueda S, Takanashi M, Sudo K, Kanekura K, Kuroda M. miR-27a ameliorates chemoresistance of breast cancer cells by disruption of reactive oxygen species homeostasis and impairment of autophagy. Lab Investig. 2020;100:863-73. https://doi.org/10.1038/s41374-020-0409-4.

83. Shi Y, Zhao Y, Shao N, Ye R, Lin Y, Zhang N, et al. Overexpression of microRNA-96-5p inhibits autophagy and apoptosis and enhances the proliferation, migration and invasiveness of human breast cancer cells. Oncol Lett. 2017;13:4402-12.

84. Meijer AJ, Lorin S, Blommaart EF, Codogno P. Regulation of autophagy by amino acids and MTOR-dependent signal transduction. Amino Acids. 2015;47:2037-63.

85. Sikder MOF, Sivaprakasam S, Brown TP, Thangaraju M, Bhutia YD, Ganapathy V. SLC6A14, a Na+/Cl--coupled amino acid transporter, functions as a tumor promoter in colon and is a target for Wnt signaling. Biochem J. 2020:477:1409-25. 
86. Wu Q, Li J, Li Z, Sun S, Zhu S, Wang L, et al. Exosomes from the tumour-adipocyte interplay stimulate beige/brown differentiation and reprogram metabolism in stromal adipocytes to promote tumour progression. J Exp Clin Cancer Res. 2019;38:223.

87. Moruno F, Pérez-Jiménez E, Knecht E. Regulation of autophagy by glucose in mammalian cells. Cells. 2012;1:372-95.

88. Wang Y, Li P, Wang S, Hu J, Chen XA, Wu J, et al. Anticancer peptidylarginine deiminase (PAD) inhibitors regulate the autophagy flux and the mammalian target of rapamycin complex 1 activity. J Biol Chem. 2012;287:25941-53.

89. Singh B, Reddy PG, Goberdhan A, Walsh C, Dao S, Ngai l, et al. p53 regulates cell survival by inhibiting PIK3CA in squamous cell carcinomas. Genes Dev. 2002;16:984-93.

90. Tang D, Kang R, Livesey KM, Cheh CW, Farkas A, Loughran P, et al. Endogenous HMGB1 regulates autophagy. J Cell Biol. 2010;190:881-92.

91. LuT, Zhu Z, Wu J, She H, Han R, Xu H, et al. DRAM1 regulates autophagy and cell proliferation via inhibition of the phosphoinositide 3-kinaseAkt-mTOR- ribosomal protein $\mathrm{S} 6$ pathway. Cell Commun Signal. 2019;7:28.

92. Meng C, Liu Y, Shen Y, Liu S, Wang Z, Ye Q, et al. MicroRNA-26b suppresses autophagy in breast cancer cells by targeting DRAM1 mRNA, and is downregulated by irradiation. Oncol Lett. 2018;15:1435-40.

93. Sun Q, Liu T, Yuan Y, Guo Z, Xie G, Du S, et al. MiR-200c inhibits autophagy and enhances radiosensitivity in breast cancer cells by targeting UBQLN1. Int J Cancer. 2015;136:1003-12.

94. Lee DY, Arnott D, Brown EJ. Ubiquilin4 is an adaptor protein that recruits Ubiquilin1 to the autophagy machinery. EMBO Rep. 2013;14:373-81. https://doi.org/10.1038/embor.2013.22.

95. Cheung CHA, Chang YC, Lin TY, Cheng SM, Leung E. Anti-apoptotic proteins in the autophagic world: an update on functions of XIAP, Survivin, and BRUCE. J Biomed Sci. 2020;27:31.

96. Chen P, He Y-H, Huang X, Tao S-Q, Wang X-N, Yan H, et al. MiR-23a modulates $X$-linked inhibitor of apoptosis-mediated autophagy in human luminal breast cancer cell lines. Oncotarget. 2017;8:80709-21.

97. Chen M, Chen Z, Wang Y, Tan Z, Zhu C, Li Y, et al. Mitophagy receptor FUNDC1 regulates mitochondrial dynamics and mitophagy.
Autophagy. 2016;12:689-702. https://doi.org/10.1080/15548627.2016. 1151580 .

98. Xu G, Shen H, Nibona E, Wu K, Ke X, Al Hafiz MA, et al. Fundc1 is necessary for proper body axis formation during embryogenesis in zebrafish. Sci Rep. 2019;9:18910.

99. Hu Q, Yuan Y, Wu Y, Huang Y, Zhao Z, Xiao C. MicroRNA-137 exerts protective effects on hypoxia-induced cell injury by inhibiting autophagy/ mitophagy and maintaining mitochondrial function in breast cancer stem-like cells. Oncol Rep. 2020:44:1627-37.

100. Hao Y, Baker D, Dijke P, Ten. TGF- $\beta$-mediated epithelial-mesenchymal transition and cancer metastasis. Int J Mol Sci. 2019;20:2767.

101. Cheng Y, Li Z, Xie J, Wang P, Zhu J, Li Y, et al. MiRNA-224-5p inhibits autophagy in breast cancer cells via targeting smad4. Biochem Biophys Res Commun. 2018;506:793-8.

102. Sharma P, Singh S. Combinatorial effect of DCA and Let-7a on triplenegative MDA-MB-231 cells: a metabolic approach of treatment. Integr Cancer Ther. 2020;19:1-11.

103. Wang J, Xie S, Yang J, Xiong H, Jia Y, Zhou Y, et al. The long noncoding RNA H19 promotes tamoxifen resistance in breast cancer via autophagy. J Hematol Oncol. 2019;12:81.

104. Lan H, Lu H, Wang X, Jin H. MicroRNAs as potential biomarkers in cancer: opportunities and challenges. Biomed Res Int. 2015;2015:125094.

105. Saliminejad K, Khorshid HRK, Ghaffari SH. Why have microRNA biomarkers not been translated from bench to clinic? Futur Oncol. 2019;15:801-3.

106. Segal M, Slack FJ. Challenges identifying efficacious miRNA therapeutics for cancer. Expert Opin Drug Discov. 2020;15:987-92. https://doi.org/10. 1080/17460441.2020.1765770.

\section{Publisher's note}

Springer Nature remains neutral with regard to jurisdictional claims in published maps and institutional affiliations.
Ready to submit your research? Choose BMC and benefit from:

- fast, convenient online submission

- thorough peer review by experienced researchers in your field

- rapid publication on acceptance

- support for research data, including large and complex data types

- gold Open Access which fosters wider collaboration and increased citations

- maximum visibility for your research: over 100M website views per year

At BMC, research is always in progress.

Learn more biomedcentral.com/submissions 